\title{
Spontaneous Cholecystocutaneous Fistula of Anterior Abdominal Wall with Subcutaneous Gallstones: About a Rare Case and Review of the Literature
}

\author{
Khalid El Haoudi*, Pierlesky Elion Ossibi, Salima Rezzouk, Mourad Oussaid, \\ Karim Ibn Majdoub Hassani, Said Ait Laalim, Imane Toughrai, Khalid Mazaz \\ Visceral Surgery Department, HASSAN II University Hospital, Fez, Morocco \\ Email: "elhaoudi.81@gmail.com
}

Received December 12, 2013; revised January 10, 2014; accepted January 18, 2014

Copyright (C) 2014 Khalid El Haoudi et al. This is an open access article distributed under the Creative Commons Attribution License, which permits unrestricted use, distribution, and reproduction in any medium, provided the original work is properly cited. In accordance of the Creative Commons Attribution License all Copyrights (C) 2014 are reserved for SCIRP and the owner of the intellectual property Khalid El Haoudi et al. All Copyright ( 2014 are guarded by law and by SCIRP as a guardian.

\begin{abstract}
Spontaneous cholecystocutaneous fistula is a rare complication of chronic calculous cholecystitis, because currently gallstones are diagnosed and treated at an early stage. This occurrence is possible even if it seems actually to be rare. We report a case of spontaneous cholecystocutaneous fistula of Anterior Abdominal Wall with subcutaneous gallstones admitted in the medical oncology unit of HASSAN II University Hospital. An 64-year-old woman, presented with a large subcutaneous abscess in the right subcostal area with swelling. An abdominal computed tomography scan showed a subcutaneous gallstone and communication between the abscess and the gallbladder. Cholecystectomy was performed and the abdominal wall abscess was drained externally. This case report demonstrates that maintaining a high degree of suspicion of this rare entity is helpful in achieving correct preoperative diagnosis, and that computed tomography scan should be performed in all cases of unexplained abdominal wall suppuration.
\end{abstract}

\section{KEYWORDS}

Gallbladder; Fistula; Gallstones; Abscess

\section{Introduction}

Spontaneous cholecystocutaneous fistula is a rare complication of gallbladder empyema without treatment. The incidence has further decreased with the advent of ressucitation and surgical treatment. Traditional treatment consists of broad-spectrum antibiotics administration, cholecystectomy and fistula drainage.

Here we present a new case with anterior abdominal wall abscess secondary to subcutaneous gallstones and we analyze different data about diagnosis, possible etiology and treatment of this entity.

\section{Case Presentation}

We present a case of a 64-year-old woman, drained twice for an abdominal wall abscess, presenting to the emer-

${ }^{*}$ Corresponding author. gency department with a swelling on the anterior abdominal wall in the right upper quadrant. The swelling had developed spontaneously. Prior to admission the area had acutely become inflamed with a purulent discharge and an abscess was diagnosed.

Transabdominal ultrasonography showed a gallbladder with abnormalities of the wall, a single gallstone impacted in the infundibulum and a fluid collection with irregular margins containing fluctuating echoes adjacent to the anterior abdominal wall of the upper right abdominal quadrant. A diagnosis of spontaneous cholecystocutaneous fistula with an abdominal purulent collection was reached.

An abdominal computed tomography scan showed a subcutaneous gallstone and communication between the abscess and the gallbladder (Figure 1).

Cholecystectomy was performed and the abdominal wall abscess was drained externally and the subcutaneous 


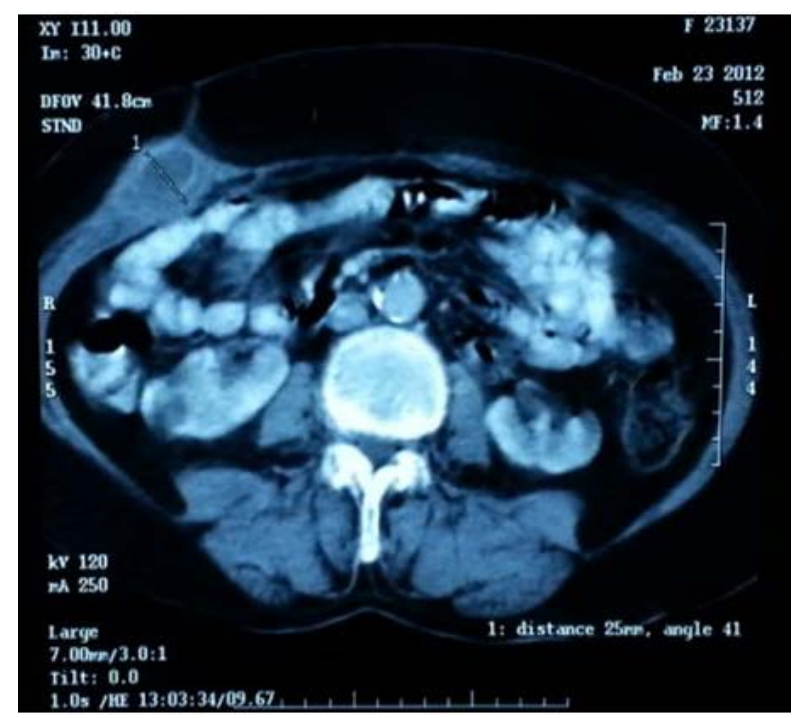

Figure 1. Abdominal CT scan: Subcutaneous gallstone with fistula between abscess and the gallbladder.

gallstone was extracted with drainage of the abdominal cavity (Figure 2).

Postoperative evolution was simple.

Anatomopathologicalstudy of the gallbladder showed no specific cholecystitis.

\section{Discussion}

Spontaneous cholecystocutaneous fistula was a common complication of gallstones until the beginning of the twentieth century and a total of 226 cases have been reported until now [1].

At present, spontaneous cholecystocutaneous fistula is a rare complication of chronic calculouscholecystitis because currently gallstones are diagnosed and treated at an early stage [2].

Spontaneous perforation of calculouscholecystitis to abdominal viscera is not uncommon, particularly to the duodenum and colon, and it has also been seen in the bronchial tree, stomach and urinary tract [3].

Like our case, most patients are females over 50 years of age, and an erythematous skin lesion may be the only presenting sign. It has been suggested that associated diseases (polyarteritesnodosa, typhoid, bacterial dissemination, and trauma) or drug treatments, such as steroids, may be predisposing factors [1].

There is usually a history of chronic biliary tract disease, however, patients generally do not report a previous distinct episode of acute cholecystitis [4].

Our patient has previous antecedent of calculousbiliary tract disease, and antecedent for abscess of right superior abdominal quadrant drained twice.

External fistulas have been reported as a result of the chronic perforation of the gallbladder, and obstruction of the cystic duct by a stone plays an important role in the

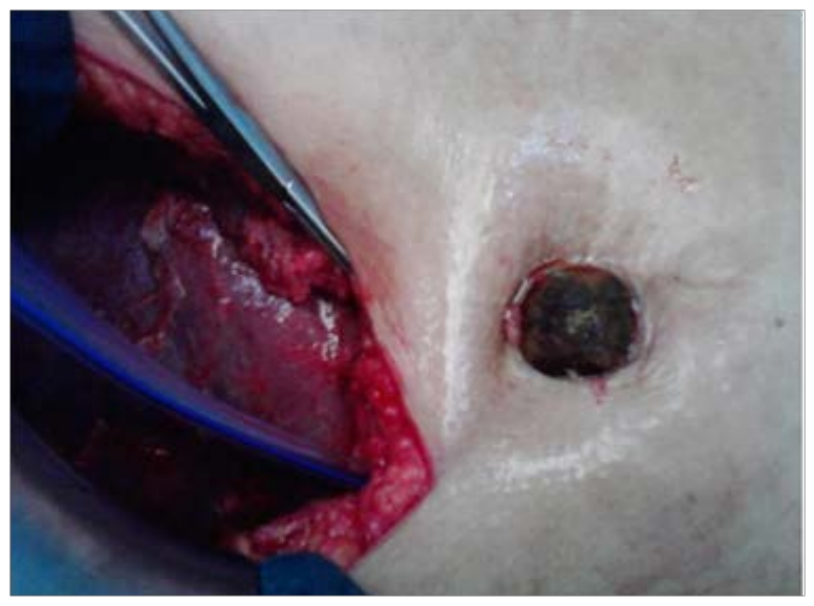

Figure 2. Operative view: Subcutaneous gallstones.

pathophysiology of perforation; adherence to the abdominal wall and necrosis of the gallbladder lead to fistula formation [5].

The clinical presentation of a cutaneous biliary fistula is variable and depends on the anatomical course; an external fistula drains more frequently in the right superior abdominal quadrant, right flank or umbilicus, even if the opening fistula tract may be present in the left-side costal margin, right-side iliac fossa, right groin or right gluteus [1].

For our patient, the clinical presentation was anterior abdominal wall abscess in the superior right quadrant with swelling.

Ultrasonography and CT are presently the best imaging modalities for diagnosing this complication [1]. Abdominal CT scan showed gallstones and communication between the abscess and the gallbladder [6].

Following control of the acute inflammatory process in the abdominal wall, an elective cholecystectomy is advisable in these patients. An open approach is usually favoured, although a laparoscopic technique has been described by Kumar [7].

\section{Conclusion}

This case underlines the fact that all patients presenting with right-sided abdominal wall abscesses need to be investigated thoroughly for underlying pathology, particularly on a background of calculous biliary tract disease.

\section{REFERENCES}

[1] R. Pezzilli, B. Barakat, R. Corinaldesi and M. Cavazzab, "Spontaneous Cholecystocutaneous Fistula," Case Reports in Gastroenterology, Vol. 4, No. 3, 2010 pp. 356360.

[2] S. Yüceyar, S. Ertürk, I. Karabiçak, E. Onur and F. Aydogan, "Spontaneous Cholecystocutaneous Fistula Presenting with an Abscess Containing Multiple Gallstones," 
a Case Report," Mount Sinai Journal of Medicine, Vol. 72, No. 6, 2005, pp. 402-404.

[3] S. Ijaz, S. Lidder, W. Mohamid and H. H. Thompson, "Cholecystocutaneous Fistula Secondary to Chronic Calculous Cholecystitis,” Case Reports in Gastroenterology, Vol. 2, No. 1, 2008, pp. 71-75. http://dx.doi.org/10.1159/000118831

[4] T. Nicholson, M. W. Born and E. Garber, "Spontaneous Cholecystocutaneous Fistula Presenting in the Gluteal Region,” Journal of Clinical Gastroenterology, Vol. 28, No. 3, 1999, pp. 276-277. http://dx.doi.org/10.1097/00004836-199904000-00024

[5] A. John, C. Murphy, D. Vimalachandran, N. Howes and
P. Ghaneh, "Anterior Abdominal Wall Abscess Secondary to Subcutaneous Gallstones," Case Reports in Gastroenterology, Vol. 2, No. 2, 2008, pp. 219-223. http://dx.doi.org/10.1159/000137375

[6] Y. Ozdemir, E. Yucel , I. Sucullu, I. Filiz, B. Gulec, M. L. Akin and M. Yildiz, "Spontaneous Cholecystocutaneous Fistula as a Rare Complication of Gallstones,” Bratislavské Lekárske Listy, Vol. 113, No. 7, 2012, pp. 445447.

[7] S. S. Kumar, "Laparoscopic Management of Cholecystocutaneous Abscess," The American Journal of Surgery, Vol. 64, No. 12, 1999, pp. 1192-1194. 